\title{
Two NADPH: Protochlorophyllide Oxidoreductase (POR) Isoforms Play Distinct Roles in Environmental Adaptation in Rice
}

\author{
Choon-Tak Kwon ${ }^{1 \dagger}$, Suk-Hwan Kim ${ }^{1 \dagger}$, Giha Song ${ }^{1}$, Dami Kim ${ }^{1}$ and Nam-Chon Paek ${ }^{1,2^{*}}$
}

\begin{abstract}
Background: NADPH: protochlorophyllide oxidoreductase (POR) is an essential enzyme that catalyzes the photoreduction of protochlorophyllide to chlorophyllide, which is ultimately converted to chlorophyll in developing leaves. Rice has two POR isoforms, OsPORA and OsPORB. OsPORA is expressed in the dark during early leaf development; OsPORB is expressed throughout leaf development regardless of light conditions. The faded green leaf $(f g l)$ is a loss-of-function osporB mutant that displays necrotic lesions and variegation in the leaves due to destabilized grana thylakoids, and has increased numbers of plastoglobules in the chloroplasts. To investigate whether the function of OsPORA can complement that of OSPORB, we constitutively overexpressed OsPORA in $\mathrm{fgl}$ mutant.

Results: In the 35S:OsPORA/fgl (termed OPAO) transgenic plants, the necrotic lesions of the mutant disappeared and the levels of photosynthetic pigments and proteins, as well as plastid structure, were recovered in developing leaves under natural long days in the paddy field and under short days in an artificially controlled growth room. Under constant light conditions, however, total chlorophyll and carotenoid levels in the developing leaves of OPAO plants were lower than those of wild type. Moreover, the OPAO plants exhibited mild defects in mature leaves beginning at the early reproductive stage in the paddy field.

Conclusions: The physiological function of OsPORB in response to constant light or during reproductive growth cannot be completely replaced by constitutive activity of OsPORA, although the biochemical functions of OsPORA and OsPORB are redundant. Therefore, we suggest that the two OsPORs have differentiated over the course of evolution, playing distinct roles in the adaptation of rice to the environment.
\end{abstract}

Keywords: Rice, Faded green leaf, OsPORA, OsPORB, Chlorophyll synthesis

\section{Background}

In angiosperms, chlorophyll (Chl) absorbs light and imparts its energy to other components of the electron transport chain during photosynthesis (Grossman et al. 1995; Barber et al. 2000). Chl is an essential compound in higher plants. However, the intermediate compounds in Chl synthesis can bind to oxygen molecules, leading to the production of reactive oxygen species (ROS)

\footnotetext{
*Correspondence: ncpaek@snu.ac.kr

${ }^{\dagger}$ Equal contributors

'Department of Plant Science, Plant Genomics and Breeding Institute, Research Institute of Agriculture and Life Sciences, Seoul National University, Seoul 08826, Republic of Korea

${ }^{2}$ Crop Biotechnology Institute, Institutes of Green Bio Science and Technology, Seoul National University, Pyeongchang 25354, Republic of Korea
}

including singlet oxygen radicals (op den Camp et al. 2003). The accumulation of ROS accelerates cellular signaling pathways or oxidizes cellular elements, leading to photo-oxidative damage and cell death (Kim et al. 2008). Therefore, Chl synthesis must be precisely controlled during chloroplast development (Sakuraba et al. 2013). During Chl synthesis, NADPH: protochlorophyllide oxidoreductases (PORs) catalyze the lightdependent conversion of protochlorophyllide (Pchlide) to chlorophyllide (Chlide), a critical intermediate step in the process (Virgin et al. 1963; Henningsen 1970; Griffiths 1978; Masuda and Takamiya 2004).

In angiosperms, during skotomorphogenesis in the dark, PORs combine with NADPH and Pchlide to produce a ternary complex that makes up the main protein 
component of the prolamellar body (PLB) in the etioplast (Schoefs and Franck 2003; Paddock et al. 2012). The PLB, which has a lattice-like structure, also contains Chl precursors, carotenoids, and lipids (Rosinski and Rosen 1972; Selstam and Sandelius 1984). The NADPH: POR: Pchlide ternary complex reduces the D-ring in Pchlide to produce Chlide, which is esterified and modified to create Chl $a$ and $\mathrm{Chl} b$ in a light-dependent manner (Apel et al. 1980; Lebedev and Timko 1998; Heyes and Hunter 2005). Through the process of light-induced greening, the PLB in the etioplast collapses and the grana thylakoids emerge, resulting in the formation of chloroplasts (Virgin et al. 1963; Henningsen 1970). This photomorphogenesis process in higher plants begins with the visible accumulation of $\mathrm{Chl}$, which is a consequence of the functions of the NADPH: POR: Pchlide ternary complex (Solymosi et al. 2007). At the same time, the transition from etioplasts containing PLBs to chloroplasts with mature thylakoids is closely associated with the role of POR in Chl synthesis (Solymosi et al. 2007). Thus, POR is ultimately involved in the formation of thylakoid membranes in higher plants (Forreiter et al. 1991).

Many gymnosperms, algae, and cyanobacteria can synthesize photosynthetically competent chloroplasts, even in the dark, because they contain two different enzymes, light-dependent POR (LPOR) and dark-operative POR (DPOR) (Forreiter and Apel 1993; Shui et al. 2009); LPOR requires light for its function, but DPOR can function in the absence of light. By contrast, angiosperms possess only LPORs; the functional deficiency of DPOR in higher plants may be related to the adaptation of specific POR regulatory mechanisms (Masuda and Takamiya 2004; Paddock et al. 2010). In addition, phylogenic analysis suggests that gene duplication might have resulted in the formation of POR families (Masuda and Takamiya 2004).

The roles of PORs have been well-studied in angiosperms, including Arabidopsis thaliana (Armstrong et al. 1995; Benli et al. 1991; Oosawa et al. 2000), Nicotiana tabacum (Zavaleta-Mancera et al. 1999; Masuda et al. 2002), Zea mays (Millerd and McWilliam 1968; Hopkins 1982; Hopkins and Elfman 1984), Avena sativa (Darrah et al. 1990), Hordeum vulgare (Schulz et al. 1989; Holtorf et al. 1995), Triticum aestivum (Teakle and Griffiths 1993), Cucumis sativus (Yoshida et al. 1995; Fusada et al. 2000), Amaranthus tricolor (Iwamoto et al. 2001), Brassica oleracea (Solymosi et al. 2004), and Pisum sativum (Spano et al. 1992). Three POR genes (AtPORA, $A t P O R B$, and AtPORC) have been identified in Arabidopsis (Armstrong et al. 1995; Oosawa et al. 2000). Whereas barley and tobacco have two isoforms of $P O R$ (Holtorf et al. 1995; Masuda et al. 2002), cucumber and pea possess only a single POR (Spano et al. 1992; Fusada et al. 2000).
The biological functions of PORs in barley and Arabidopsis are well known, as these plants are representative monocots and dicots, respectively. The two POR isoforms in barley are distinct, although the enzymatic activities of HvPORA and HvPORB in vitro are similar (Holtorf et al. 1995). For example, HvPORA is mainly expressed in etiolated seedlings and is downregulated by illumination, but $H v P O R B$ is constitutively expressed during leaf development, regardless of light conditions (Holtorf et al. 1995). In Arabidopsis, the functions and expression patterns of AtPORA and AtPORB are analogous to those of the two PORs in barley (Armstrong et al. 1995; Runge et al. 1996). However, AtPORC is upregulated in the light and downregulated in the dark (Oosawa et al. 2000; Su et al. 2001). Moreover, AtPORC expression strongly increases under high light conditions, indicating that AtPORC activity is crucial for protecting $\mathrm{Chl}$ from breakdown under excessive light during plant development (Masuda et al. 2003).

Rice has two POR isoforms, OsPORA and OsPORB. A mutation in OsPORB (osporB) was first identified in a study of the faded green leaf $(f g l)$ mutant, which displays necrotic lesions and variegation during leaf development (Sakuraba et al. 2013). The defects in $f g l$ mutant are strongly associated with the reduced expression of OsPORA and the lack of OsPORB activity in developing leaves (Sakuraba et al. 2013). Also, the formation of ROS, which is induced by the accumulation of nonphotoactive Pchlide, causes the necrotic phenotype in the $f g l$ mutant leaves (Chakraborty and Tripathy 1992; op den Camp et al. 2003; Sakuraba et al. 2013). The biological functions of OsPORA and OsPORB are highly similar to those of HvPORA and HvPORB, respectively (Sakuraba et al. 2013). In addition, OsPORB is upregulated under high light treatment, suggesting that the function of OsPORB overlaps with that of AtPORC in Chl synthesis and maintenance (Sakuraba et al. 2013).

Although some species, including cucumber and pea, have only one POR, many photosynthetic plants have two or three isoforms of POR. Therefore, por mutants must be identified and characterized in every angiosperm to explore the biological diversity of POR members in detail, for example by studies of Arabidopsis POR isoforms (Frick et al. 2003; Masuda et al. 2003; Paddock et al. 2012; Sakuraba et al. 2013). In addition to studying por mutants, the functional redundancy of PORs has been examined by producing genetically modified Arabidopsis plants. Constitutive overexpression of AtPORA can restore the phenotypes of the atpor $B$ atpor $C$ double mutant (Paddock et al. 2010). In the current study, based on this finding, we explored whether OsPORA can fully substitute for OsPORB by producing transgenic rice plants constitutively overexpressing OsPORA in the $f g l$ mutant background. We 
analyzed the transgenic plants under various growth conditions, finding that the function of OSPORB can be replaced by constitutively expressing OsPORA during vegetative growth under normal long days in paddy field conditions, but not under excessive light conditions or during reproductive growth. We discuss the physiological and developmental differentiation of the two rice PORs.

\section{Results}

Constitutive Overexpression of OsPORA in fgl Mutant Background Rescues Chl Synthesis and Leaf Development under Natural Field Conditions

To examine the functional redundancy between OsPORA and OsPORB, we introduced OsPORA cDNA fused to the cauliflower mosaic virus $35 \mathrm{~S}$ promoter (35S:OsPORA) into $f g l$ mutant by Agrobacterium-mediated transformation and generated 97 independent 35S:OsPORA/fgl (hereafter, OPAO) transgenic $\mathrm{T}_{0}$ lines (Fig. 1a). Most OPAO plants exhibited normal leaf development under natural sunlight in the greenhouse compared with $f g l$ mutant (data not shown). Among the OPAO lines, we selected three lines with different phenotypes based on leaf color: deep green (line \#11), intermediate green (line \#2), and light green (line \#27) (Fig. 1b and c). Genomic PCR using specific primers, including regions of the $35 S$ promoter and OsPORA, amplified a single genomic DNA fragment from the transgenic lines (Additional file 1: Figure S1). We confirmed the expression levels of OsPORA mRNA in mature leaves of the transgenic lines. OsPORA is more highly expressed in the light green sectors of $f g l$ leaves than in wild type (WT) (Sakuraba et al. 2013). We found the level of OsPORA mRNA in $f g l$ mutant was approximately three times that of WT, and the mRNA levels of the three transgenic lines were over 100-fold higher than that of $f g l$ mutant (Fig. 1d). In particular, the transcript level in line \#11 was approximately 280-fold higher than that of $f g l$ mutant (Fig. 1d). The following year, we generated $T_{1}$ progeny from three transgenic $T_{0}$ lines. The $T_{1}$ plants were selected on the growth medium including hygromycin and transferred to a paddy field. The OsPORA mRNA levels of $\mathrm{T}_{1}$ plants (Additional file 2: Figure S2) were similar to those of $\mathrm{T}_{0}$ lines (Fig. 1d). The following year, homozygous $\mathrm{T}_{2}$ plants were selected by progeny testing on growth medium with hygromycin. The homozygous $\mathrm{T}_{2}$ progeny of line \#11 were grown in the paddy field and showed a phenotypically rescued green leaf color, similar to WT (Additional file 3: Figure S3). These results suggest that overexpressing OsPORA can recover the defective leaf color phenotype of $f g l$ mutant under natural growth conditions.

OsPORA Overexpression Restores Photosynthetic Proteins and Pigments and Inhibits ROS Production in fgl Mutant Next, we measured photosynthetic pigments in the leaf tissues of WT, $f g l$, and OPAO plants. Total Chl concentrations increased and the $\mathrm{Chl} a / b$ ratio decreased in the three OPAO lines compared to $f g l$ mutant (Fig. 1e and f). The Chl level in OPAO line \#11 was similar to that of WT, indicating that constitutive expression of OsPORA fully recovered Chl synthesis in $f g l$ mutant background. Moreover, the total carotenoid content was fully recovered in line \#11 (Fig. 1g). Similarly, the photosynthetic protein levels were higher in the OPAO lines than in $f g l$ mutant (Fig. 1h). Thus, the levels of Chl synthesis and the formation of the photosynthetic apparatuses in the OPAO lines were nearly proportional to the expression levels of OsPORA. These results strongly suggest that OsPORA can substitute for OsPORB in Chl synthesis if OsPORA is constitutively expressed in developing leaves under natural growth conditions.

Yellow/white leaf variegation in $f g l$ mutant is induced by excessive accumulation of ROS such as singlet oxygen $\left({ }^{1} \mathrm{O}_{2}\right)$ (Sakuraba et al. 2013). We thus examined the levels of singlet oxygen in the OPAO lines. In contrast to $f g l$ mutant, singlet oxygen did not accumulate in the three OPAO lines (Fig. 1i), suggesting that the recovery of the necrotic lesion phenotype in these lines is related to the decrease in ROS.

\section{OPAO Plants Exhibit Normal Chloroplast and Etioplast Structure}

In $f g l$ mutant, the chloroplasts in both light green sectors and yellow/white sectors of $f g l$ leaves display defective thylakoid stacking and have plastoglobules (Sakuraba et al. 2013). To investigate the chloroplast structure in OPAO plants, we sampled 80-day-old leaves from OPAO line \#11 (Fig. 2a-c). The chloroplasts in the yellow/white sectors of $f g l$ mutant were damaged and contained many plastoglobules and unstacked grana thylakoids (Fig. 2b). By contrast, the chloroplasts of WT and line \#11 contained well-stacked grana thylakoids without plastoglobules (Fig. 2a and c), suggesting that the recovery of the leaf phenotype in OPAO plants is closely associated with the rescue of chloroplast development. Moreover, the restoration of thylakoid stacking by OsPORA overexpression might be related to the increased $\mathrm{Chl}$ concentration in $f g l$ mutant (Fig. 1e). POR is involved in the production of prolamellar bodies (PLBs), which include carotenoids and Chl precursors in the etioplast (Rosinski and Rosen 1972; Engdahl et al. 2001), as the sizes of PLBs are reduced in the etioplasts of por mutants in both Arabidopsis and rice (Frick et al. 2003; Masuda et al. 2003; Paddock et al. 2012; Sakuraba et al. 2013). To examine whether PLBs are larger in the OPAO lines than in $f g l$, we observed the ultrastructures of etioplasts in 10-day-old $\mathrm{T}_{2}$ seedlings grown in dark conditions. The PLBs were much larger in OPAO line \#11 than in $f g l$ mutant but similar to those of WT (Fig. 2d-g). PLBs from line \#11 also exhibited a well-organized lattice structure like 


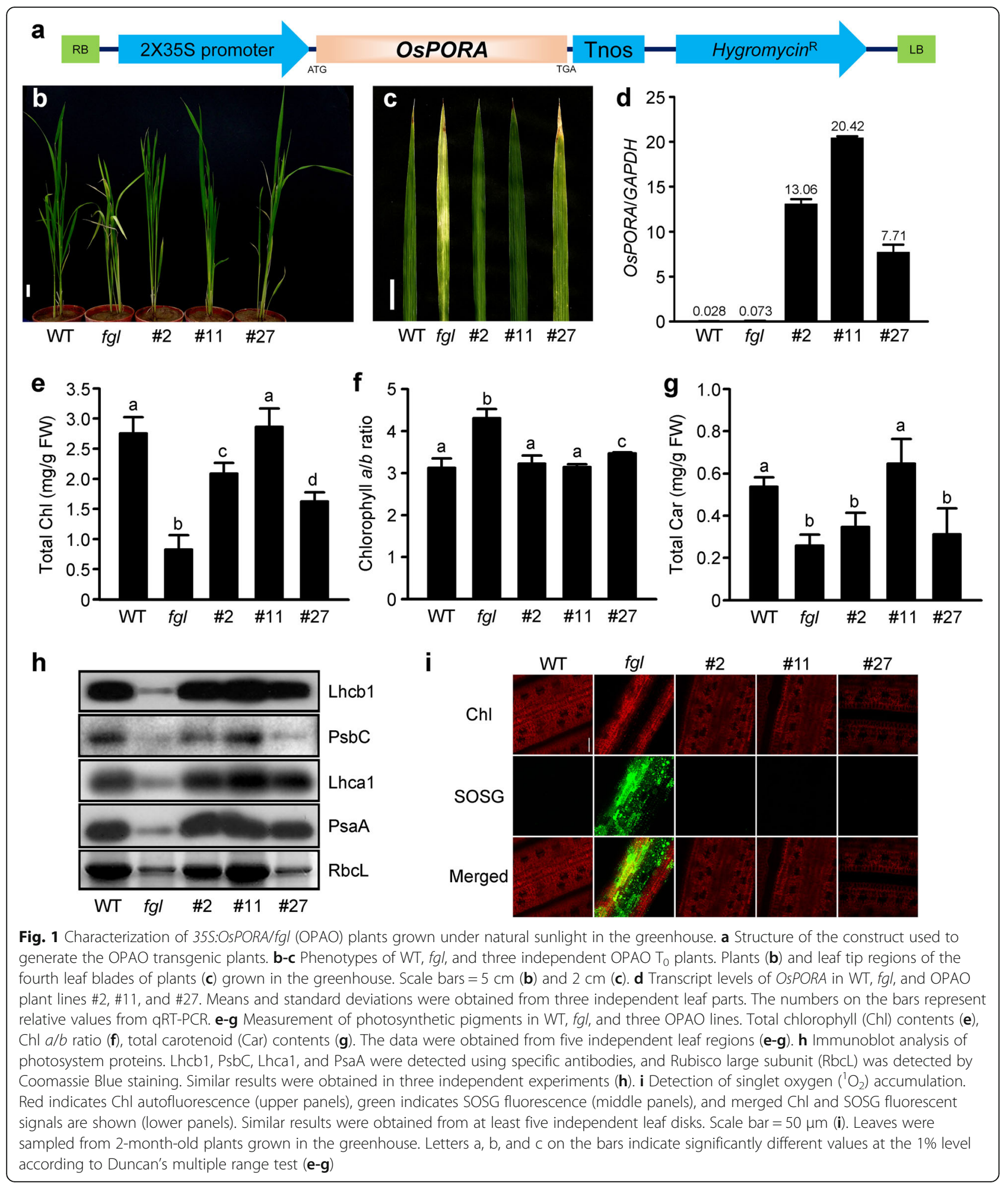

that of WT (Fig. 2d and g). These results demonstrate that constitutive overexpression of OsPORA can fully recover the function of OsPORB during both etioplast and chloroplast development in developing and mature leaves in the absence of OsPORB activity.
Overexpression of OsPORA Restores Normal Total and Photoactive Pchlide Levels

Several studies in Arabidopsis and rice have suggested that PLB size is related to the levels of total and photoactive Pchlides (Franck et al. 2000; Paddock et al. 2010; 

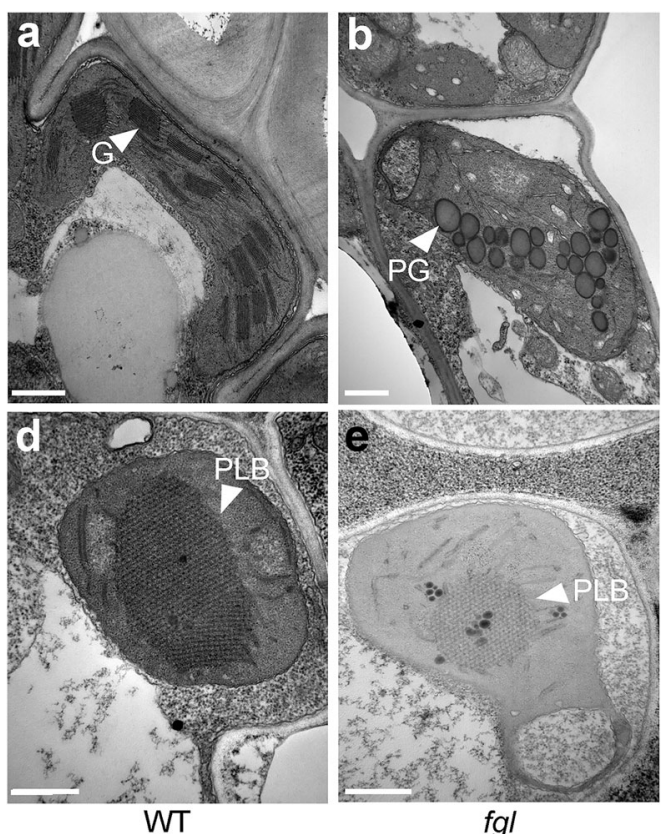

fgl

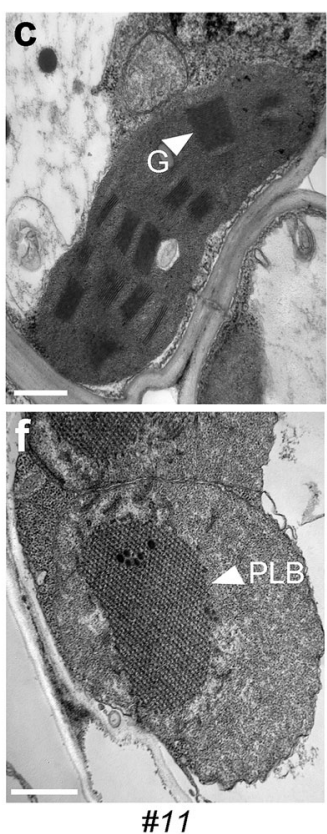

Fig. 2 Ultrastructural analysis of plastids in the leaves of WT, fgl, and OPAO plants. a-c Chloroplasts in the WT leaf (a), the yellow sector of an fgl leaf (b), and the OPAO \#11 homozygous $T_{2}$ line leaf (c) grown for 80 days in the greenhouse. G, grana thylakoid; PG, plastoglobule. d-f Etioplasts in etiolated leaves of WT (d), fgl (e), and OPAO \#11 line (f) grown in the dark for 10 days. PLB, prolamellar body. Scale bars $=0.5 \mu m(\mathbf{a}-\mathbf{f})$. Similar results were obtained in at least three independent samples

Sakuraba et al. 2013). In particular, the levels of total and photoactive Pchlides in $f g l$ mutant are significantly lower than those in WT, which is consistent with the reduced size of PLBs in $f g l$ mutants (Sakuraba et al. 2013). We therefore collected 10-day-old etiolated WT, $f g l$, and OPAO line \#11 seedlings and measured their total and photoactive Pchilde levels using fluorescence and UV/ VIS spectrophotometry (Fig. 3 and Additional file 4: Figure S4). As previously shown, both total and photoactive Pchlide levels were markedly reduced in $f g l$ mutant compared to WT (Fig. 3). However, the concentrations of total and photoactive Pchlides in OPAO line \#11 increased to the WT levels (Fig. 3). Furthermore, the ratio of photoactive to total Pchlides was approximately 0.63 in WT, 0.45 in $f g l$, and 0.66 in line \#11, suggesting that the photoactive Pchlide level was completely recovered in etiolated seedlings of the OPAO line. We obtained similar results from UV/VIS spectrophotometry of Pchlide levels in WT, $f g l$, and line \#11 plants (Additional file 4: Figure S4). These data suggest that in the absence of OsPORB, OsPORA overexpression leads to the formation of photoactive Pchlide for normal Chl synthesis during very early rice development.

\section{OPAO Plants Show Partially Rescued Chl and Carotenoid Contents under Constant Light Conditions}

OsPORA is dark-induced and expressed at early stages of leaf development; also, OsPORA contributes to light- dependent $\mathrm{Chl}$ synthesis and inhibits the formation of necrotic lesions during early leaf development in the presence of OsPORB (Sakuraba et al. 2013). Under constant light (CL) conditions, fgl mutant exhibited severe leaf variegation and repressed Chl synthesis (Sakuraba et al. 2013); the rapid decrease in OsPORA expression may be the main reason for the defective phenotype. Thus, to investigate whether constitutive overexpression of OSPORA could compensate for the effects of OsPORB deficiency on Chl synthesis under CL conditions, we grew WT, $f g l$, and line \#11 plants for 2 weeks under CL conditions and determined their leaf phenotypes and levels of photosynthetic pigments. Twoweek-old OPAO line \#11 plants had normal green leaves with no necrotic lesions, which is similar to the WT phenotype, indicating that ectopic expression of OsPORA rescues leaf development in the absence of OsPORB, even under CL conditions (Fig. 4a).

Next, we analyzed the phenotypes of the other OPAO lines (line \#2 and \#27; Fig. 1) in more detail. The three OPAO lines had different levels of OsPORA mRNA (Fig. 1d and Additional file 2: Figure S2) and displayed dark (line \#11), intermediate (line \#2), and light green (line \#27) leaf color (Fig. 4b). In line \#27, which had the lowest OsPORA mRNA level among OPAO lines, the leaf color and photosynthetic protein levels were similar to those of $f g l$ mutant (Fig. $4 \mathrm{~b}$ and c). We also examined total Chl contents, $\mathrm{Chl} a / b$ ratios, and total carotenoid 

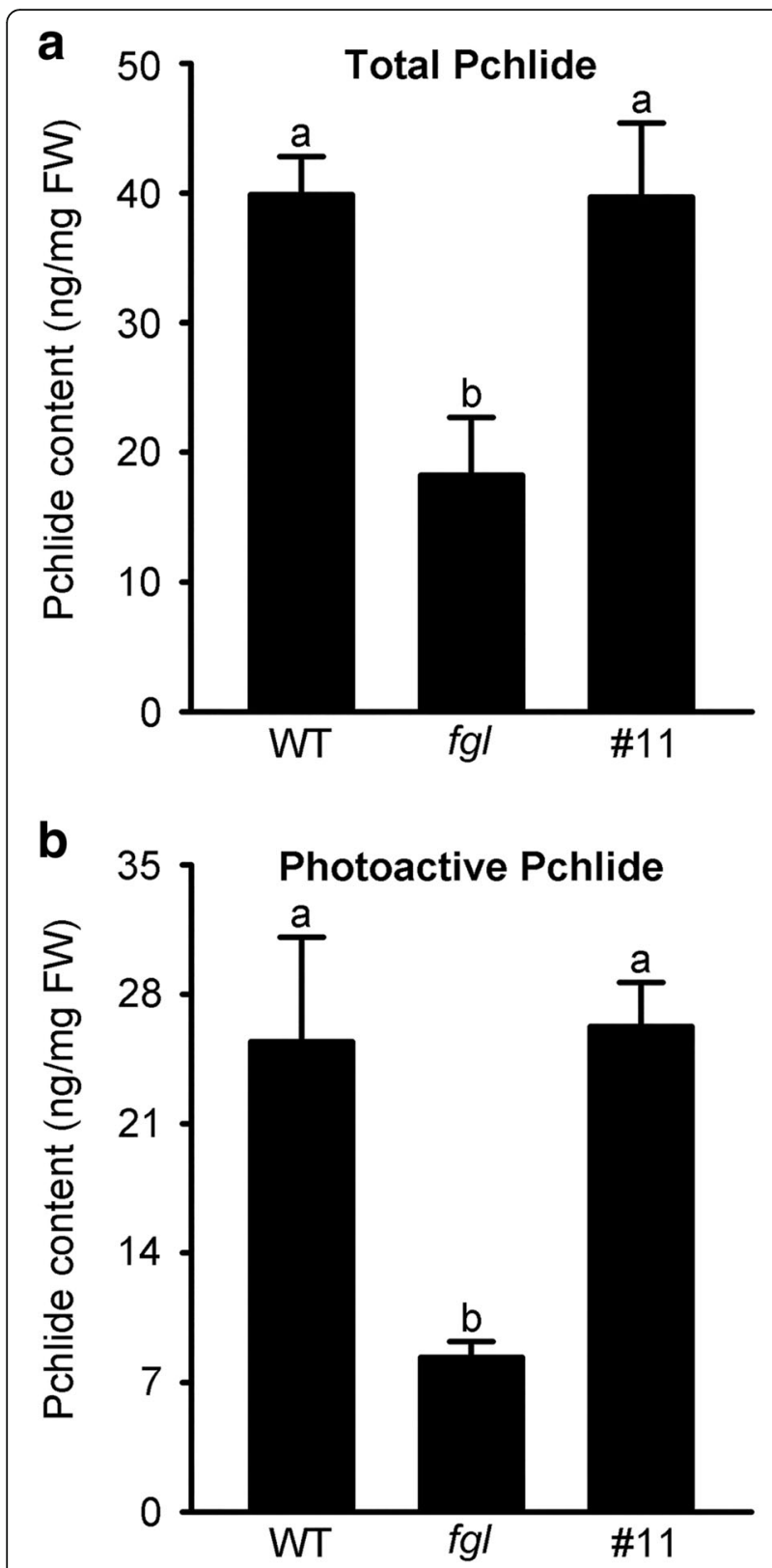

Fig. 3 Levels of total Pchlide and photoactive Pchlide in WT, fgl, and the OPAO line. a Total Pchlide was measured based on fluorescence emission in pigments extracts from 10-day-old etiolated seedling grown in darkness. b Photoactive Pchlide levels were calculated by subtracting non-photoactive Pchlide from total Pchlide levels, which were measured in light-treated seedlings. The results were obtained and calculated from 10 independent etiolated leaves. The experiments were performed twice by fluorescence spectrophotometry, producing similar results. Letters $a$ and $b$ on the bars denote statistically significant differences based on Duncan's test $(P<0.01)$

levels in leaves (Fig. 4d-f). In contrast to the results under natural conditions, the total $\mathrm{Chl}$ and carotenoid contents in the three OPAO lines did not recover to WT levels (Fig. 4d and f). In particular, the photosynthetic pigment levels in line \#27 were similar to those in $f g l$ mutant, indicating that OsPORA expression in line \#27 is below the threshold level required to recover Chl synthesis in the $f g l$ mutant background (Fig. $4 \mathrm{~d}-\mathrm{f}$ ). Interestingly, in line \#11, with the highest OsPORA mRNA level, the $\mathrm{Chl}$ and carotenoid levels did not fully recover to WT levels (Fig. 4d and f). We then performed phenotypic characterization of line \#11 plants grown under short day (SD) conditions compared to CL conditions (Additional file 5: Figure S5). Under SD conditions, all leaf-associated phenotypes of line \#11 completely recovered to those of WT, in contrast to the results under CL conditions (Additional file 5: Figure S5). This result strongly suggests that $\mathrm{Chl}$ synthesis in the absence of OsPORB cannot be completely restored during early development under CL conditions, even though OsPORA mRNA levels are extraordinarily high.

Abnormally high levels of ROS cause the lesion formation and leaf variegation phenotypes in $f g l$ mutant (Sakuraba et al. 2013). Some leaves in line \#27 showed variegation and contained ROS such as hydrogen peroxide $\left(\mathrm{H}_{2} \mathrm{O}_{2}\right)$ and superoxide anion radicals $\left(\mathrm{O}_{2}^{-}\right)$, as was observed in the leaf tissues of $f g l$, but this was not the case in lines \#11 and \#2 (Fig. 4g and h). The leaf variegation of line \#27 under CL conditions is likely caused by ROS accumulation to levels corresponding to those found in $f g l$ mutants.

OsPORA Expression in OPAO Plants is Consistent under $\mathrm{CL}$ and SD Conditions

The expression of OsPORA decreases markedly under $\mathrm{CL}$ and high-light conditions. In addition, OsPORA protein levels are proportional to its transcript levels under various light conditions, indicating that OsPORA protein levels are regulated at the transcriptional level (Sakuraba et al. 2013). We therefore investigated whether the compromised rescue of the OPAO lines is related to changes in OSPORA mRNA or OsPORA protein levels. We grew the OPAO plants under SD and CL conditions and analyzed their OsPORA mRNA and OsPORA protein levels (Fig. 5). In contrast to our hypothesis, OPAO line \#11 showed almost the same levels of OsPORA mRNA and OsPORA protein under both SD and CL conditions (Fig. 5b and c). Furthermore, the levels of OsPORA protein in OPAO line \#11 were consistent with its transcriptional pattern (Fig. $5 \mathrm{~b}$ and c). These results indicate that the OSPORA mRNA and OsPORA protein levels in this line are not dependent on photoperiod.

\section{Recovery of the Expression of Photosynthesis- and Chl Synthesis-Related Genes in the OPAO Lines}

Several photosynthesis- and Chl synthesis-associated genes were previously found to be downregulated in the green sectors of 2-week-old $f g l$ leaves under CL conditions (Sakuraba et al. 2013). To investigate the molecular 

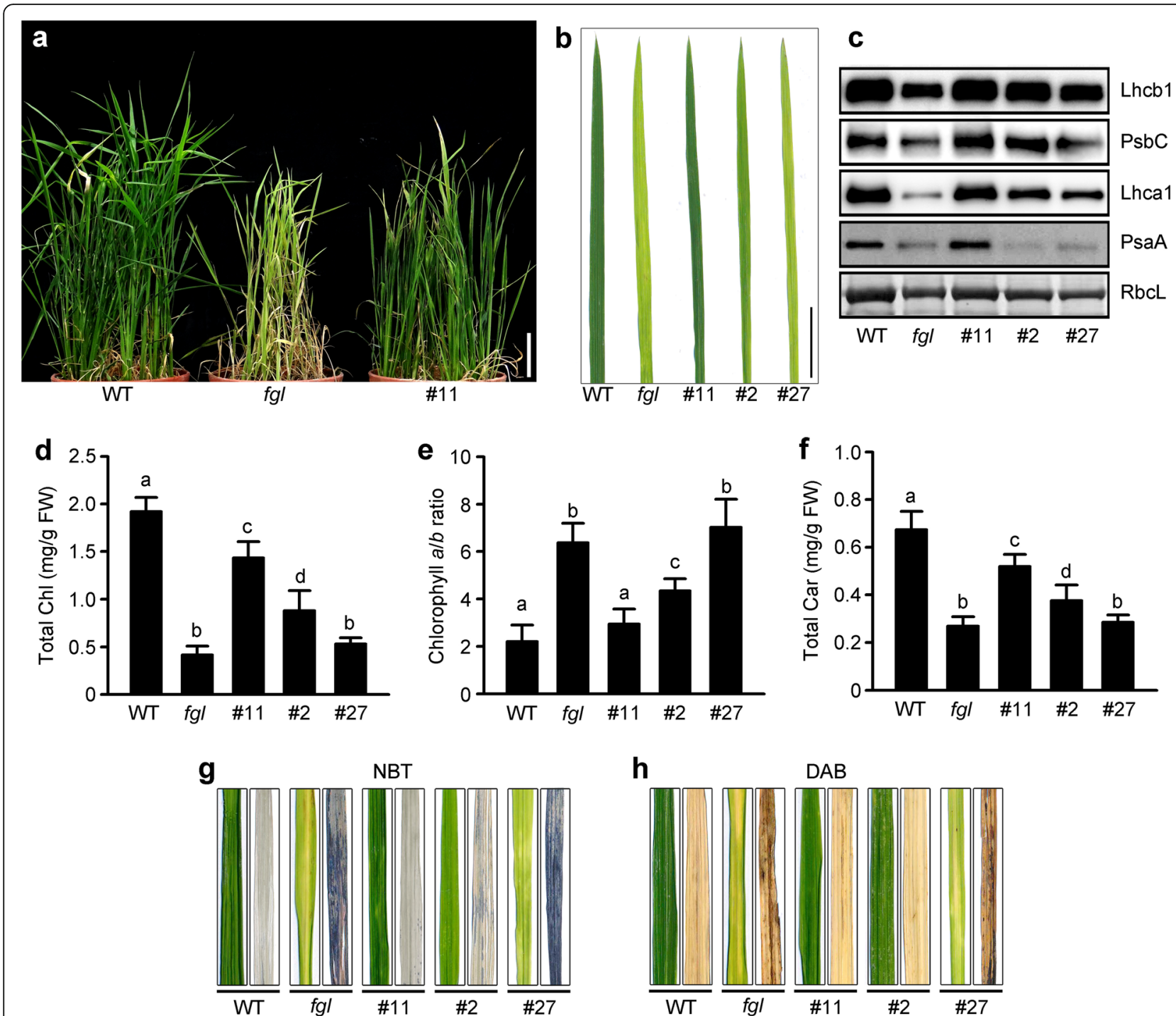

Fig. 4 Characterization of OPAO plants under $C L$ conditions. a-b Phenotypes of WT, fgl, and OPAO homozygous $T_{2}$ plants. Shoots (a) and third leaf blades (b) grown under CL conditions in an artificial growth chamber $\left(200 \mu \mathrm{mol} \mathrm{m} \mathrm{m}^{-2}\right)$. Scale bar $=2 \mathrm{~cm}$ (b). c Immunoblot analysis of photosystem proteins. The proteins were detected using specific antibodies, but RbcL was visualized by Coomassie Blue staining (c). The detection was repeated three times with independent biological replicates (c). d-f Quantification of photosynthetic pigments in WT, fgl, and OPAO line \#11. Total $\mathrm{Chl}$ contents (d), Chl a/b ratio (e), total Car contents (f). The data were obtained from 10 independent leaf samples, and means with different letter are significantly different (Duncan's test, $P<0.01)(\mathbf{d}-\mathbf{f})$. $\mathbf{g}$-h Determination of ROS accumulation in leaves. Superoxide anion radicals $\left(\mathrm{O}_{2}^{-}\right)(\mathbf{g})$ and hydrogen peroxide $\left(\mathrm{H}_{2} \mathrm{O}_{2}\right)(\mathbf{h})$ in leaves. Superoxide anion radicals and hydrogen peroxide were visualized by staining with NBT $(\mathbf{g})$ and DAB $(\mathbf{h})$, respectively. Samples were obtained from 2-week-old plants grown in a growth chamber. All analyses were repeated at least twice and showed similar results

basis for the recovery of the OPAO lines, we examined the transcriptional patterns of two photosynthesisrelated genes (Lhcb1 and $L h c b 4)$ and three genes involved in Chl synthesis (CHLH, GSAT, and DVR) in the leaves of plants grown under CL conditions (Fig. 6). The expression levels of the five genes were higher in OPAO line \#11 than in $f g l$ mutant, indicating that leaf recovery in the OPAO lines is closely associated with an increase in the expression levels of these genes (Fig. 6). However, the expression of $L h c b 1$ and $L h c b 4$ was not restored to the WT levels (Fig. 6c-e). Perhaps the partial upregulation of these genes is associated with the incomplete rescue of the OPAO lines under CL conditions.

Compromised Rescue of OPAO Plants under Natural Field Conditions Beginning at the Early Reproductive Phase

Overexpressing AtPORA in the Arabidopsis atporB atpoc $C$ double mutant fully restores $\mathrm{Chl}$ synthesis during plant growth (Paddock et al. 2010). However, we detected degreening of leaf tips in OPAO plants beginning 


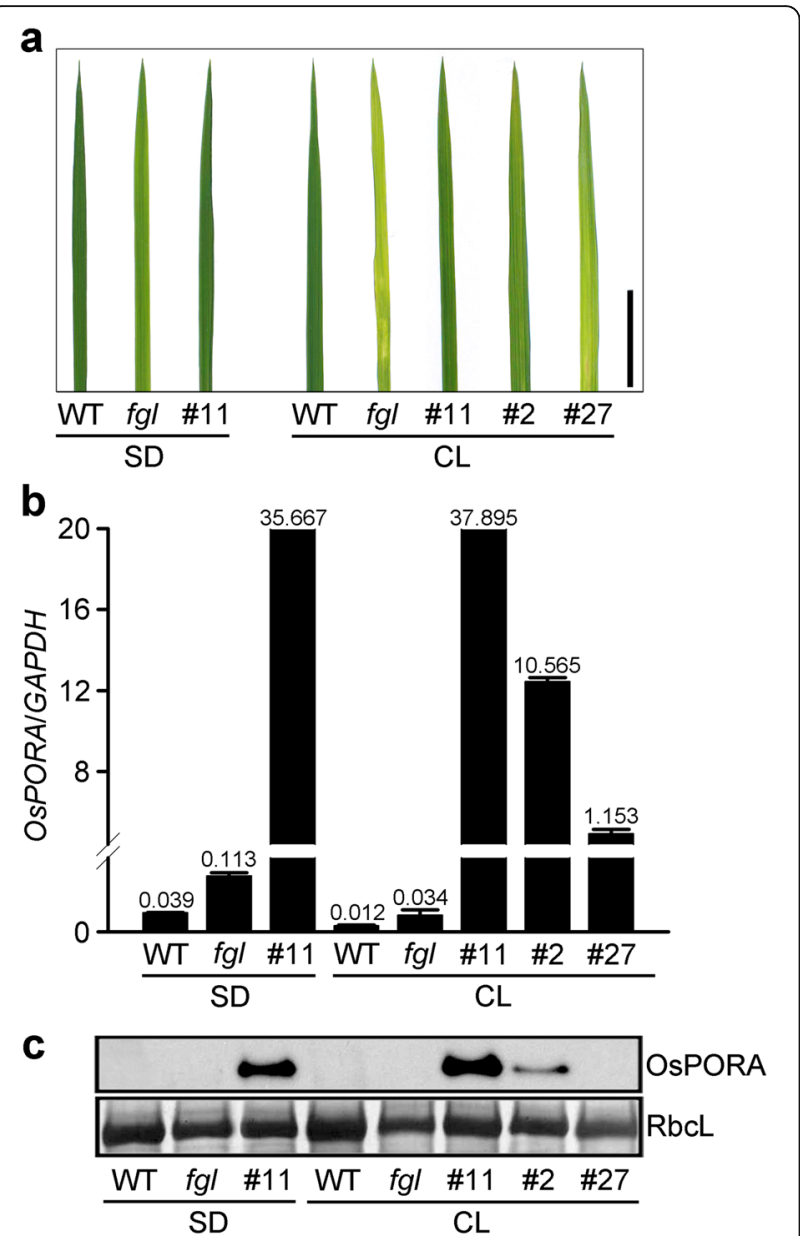

Fig. 5 Expression patterns of OsPORA transcript and OsPORA protein in $\mathrm{WT}, \mathrm{fgl}$, and OPAO lines under $\mathrm{SD}$ and $\mathrm{CL}$ conditions. a Phenotypes of third leaf blades. Plants were grown for 2 weeks in artificial growth chambers $\left(200 \mu \mathrm{mol} \mathrm{m} \mathrm{m}^{-2} \mathrm{~s}^{-1}\right)$. Scale bar $=2 \mathrm{~cm}(\mathbf{a})$. b Transcript levels of OsPORA in WT, fgl, and OPAO plants. Numbers on the bars indicate relative values quantified by RT-qPCR. Means and standard deviations were obtained from three independent leaf blades (b). c Immunoblot assay of OsPORA protein. RbcL was visualized using CCB staining as a control (c). All analyses were repeated at least twice and produced similar results

at the early reproductive stage. The distal regions of leaf blades in OPAO line \#11 plants grown in a natural paddy field turned light green beginning at 90 days after sowing (Fig. 7a). This phenomenon became severe until heading and flowering (Fig. 7a-d). The degreening process began in older leaves of $f g l$ mutant, and OPAO leaves showed an intermediate phenotype between WT and $f g l$ (Fig. $7 \mathrm{~d}$ ). We analyzed photosynthetic pigment levels in the leaves of WT, $f g l$, and line \#11 plants (Fig. 7e-g). Total $\mathrm{Chl}$ and carotenoid contents were higher in the OPAO line than in $f g l$ mutant and lower than in WT (Fig. 7e and g), which is consistent with their leaf phenotypes (Fig. 7d). In addition, the Chl $a / b$ ratio in line \#11 was higher than that of WT, as was that in $f g l$ mutant (Fig. 7f). These results suggest that OsPORA activity can compensate for the absence of OsPORB activity during the juvenile and vegetative stages of plant growth. However, after the vegetative phase, the role of OsPORA in Chl synthesis is limited, even if OsPORA is constitutively overexpressed.

\section{Discussion}

In the model dicot plant Arabidopsis, three homologous $P O R$ genes encode structurally similar enzymes that are synthesized as pro-proteins and modified in the plastid (Franck et al. 2000; Frick et al. 2003; Masuda et al. 2003; Paddock et al. 2010; Reinbothe et al. 2015). Even though the sequences of PORs are highly conserved, the mature POR isoforms play somewhat distinct roles during development due to their dissimilar expression patterns (Franck et al. 2000; Frick et al. 2003; Masuda et al. 2003; Paddock et al. 2010; Paddock et al. 2012). Rice contains two POR proteins, OsPORA and OsPORB, which have distinct functions during leaf growth. For example, OsPORA functions in the early stage of leaf development, while OsPORB functions throughout development (Sakuraba et al. 2013). In the current study, by producing transgenic rice plants overexpressing OsPORA in the $f g l$ mutant background, we found that the functional deficiency of OSPORB could be overcome by the ectopic expression of OsPORA. Under CL conditions, however, the Chl contents of the OPAO lines during early development were lower than those of WT (Fig. 4d), in contrast to the results obtained under SD conditions in growth chambers (Additional file 5: Figure $\mathrm{S} 5 \mathrm{e}$ ) or natural long day conditions in the greenhouse (Fig. 1e). Because the expression levels of OsPORA mRNA and protein in OPAO line \#11 were highly upregulated under both SD and CL conditions (Fig. 5b and c), the partial rescue of the mutant phenotype under CL conditions might be related to the lack of OsPORB, indicating that functional variation exists between OsPORA and OsPORB.

The Arabidopsis porA mutants show severe deficiencies in photoautotrophic development, as well as diminished photoactive Pchlide conversion and reduced prolamellar body volume during skotomorphogenesis (Paddock et al. 2012). In addition, the elimination of AtPORA activity during photomorphogenesis results in reduced total Chl contents and abnormal plant growth, although AtPORA is transiently expressed during illumination (Armstrong et al. 1995; Paddock et al. 2012). However, the function of OsPORA in rice is still unclear, since no reports about ospor $A$-deficient mutants are currently available. The rice $f g l$ mutant shows milder defects than Arabidopsis atporB atpor $C$ double mutants (Frick et al. 2003; Sakuraba et al. 2013). Because OsPORA is highly expressed in developing leaves at the 


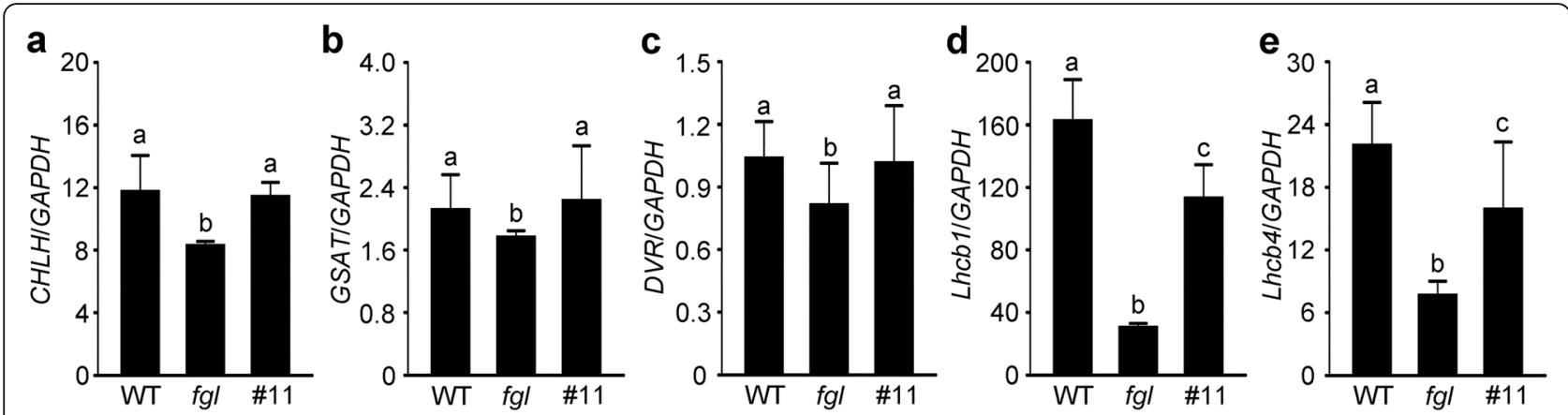

Fig. 6 Transcriptional analysis of Chl synthesis- and photosynthesis-related genes in WT, fgl, and OPAO plants. a-e Relative transcript levels of CHLH (a), GSAT (b), DVR (c), Lhcb1 (d), and Lhcb4 (e) in leaf blades of plants grown under CL conditions for 2 weeks. CHLH encodes Mg-chelatase H subunit (Os03g20700); GSAT, glutamate-1-semialdehyde aminotransferase (Os08g41990); DVR, 3,8-divinyl chlorophyllide a 8-vinyl reductase (Os03g22780); Lhcb1, light-harvesting Chl a/b-binding protein of photosystem II (Os01g41740); Lhcb4 (Os07g37240). Averages and standard deviations were determined using three biological replicates. Lower-case letters on the bars denote significantly different values determined by Duncan's multiple range test $(P<0.01)$. The experiments were conducted at least twice, showing similar results

early seedling stage, the function of OsPORA is considered to be essential for Chl synthesis in rice leaves (Sakuraba et al. 2013). Our observation of OPAO lines suggests that OsPORA functions in Chl synthesis in leaves, even after early development (Fig. 1 and Additional file 3: Figure S3). Our findings imply that the role of OsPORA in rice development is generally controlled by its expression pattern. However, OPAO $\mathrm{T}_{2}$ plants grown under natural field conditions exhibited partially recovered phenotypes in leaf blades beginning at the booting stage (before the heading stage), i.e., approximately 90 days after sowing (Fig. 7a). Finally, the leaf phenotype of the OPAO lines was intermediate between WT and $f g l$ mutant (Fig. $7 \mathrm{~d}-\mathrm{g}$ ), suggesting that beginning at the reproductive phase, Chl synthesis is not completely controlled only by OsPORA, although OsPORA expression levels are quite high. Previous and current findings suggest that the difference between the activities of OsPORA and OsPORB might at least be partially attributed to the different spatial and temporal expression patterns of the two OsPORs, as well as their differing protein functions.

A reduction in PLB size leads to reduced total Pchlide levels (Franck et al. 2000; Sakuraba et al. 2013). The PLB size in the OPAO lines was recovered to that of WT, leading to an increase in total Pchlide contents (Fig. 2d$\mathrm{g}$ and $3 \mathrm{a})$. In addition to increased total Pchlide levels, photoactive Pchlide in the OPAO lines accumulated to WT levels (Fig. 3b). This result indicates that although maintaining threshold levels of Pchlide formation in etiolated seedlings appears to require OsPORB activity, ectopic expression of OsPORA is sufficient for rescuing total and photoactive Pchlide levels in $f g l$ mutant. In other words, normal levels of OsPORA in the absence of OsPORB activity are not sufficient for the formation of enough Pchlide to maintain persistent green coloration in mature leaves. Thus, the enzymatic functions of the two OsPORs are redundant during the juvenile growth stage. Moreover, POR-mediated conversion from Pchlide to Chlide is completely light dependent (Griffiths 1978). Pchlide bound to POR is referred to as "photoactive", whereas Pchlide that is not bound by POR, which is referred to as "non-photoactive", readily functions as a photosensitizer to produce singlet oxygen, thereby inducing photo-oxidative damage (Matringe et al. 1989; Reinbothe et al. 1996; Sperling et al. 1997; Mock et al. 1998; Heyes and Hunter 2005). In addition, HvPORA plays a photo-protective role during greening through the reconstitution of the light-harvesting POR: Pchlide complex (Buhr et al. 2008). The leaf variegation in $f g l$ mutant might be caused by photosensitization induced by the accumulation of high levels of non-photoactive Pchlide (Sakuraba et al. 2013). Therefore, the rescue of necrotic lesions in the OPAO lines is closely related to the disappearance of ROS, including singlet oxygen, generated from non-photoactive Pchlide (Figs. 1i and 4g-h). In addition, ROS accumulation in $f g l$ chloroplasts likely provokes the downregulation of $\mathrm{Chl}$ synthesis and photosynthetic genes in the nucleus via retrograde signaling from the chloroplast (Gadjev et al. 2006; Sakuraba et al. 2013). Indeed, the transcript levels of photosynthesis-associated genes (Lhcb1 and Lhcb4), as well as upstream (GSAT and $C H L H)$ and downstream $(D V R)$ genes of POR, were rescued in OPAO line \#11 (Fig. 6). These results suggest that the recovery of necrotic lesions in the OPAO lines is caused by the disappearance of ROS derived from the reduction in non-photoactive Pchlide levels due to OsPORA overexpression, which upregulates these genes, thereby leading to the removal of oxidative damage.

OsPORA and OsPORB protein levels are directly proportional to their transcript levels, suggesting that OsPOR activity is mainly controlled at the transcriptional level 


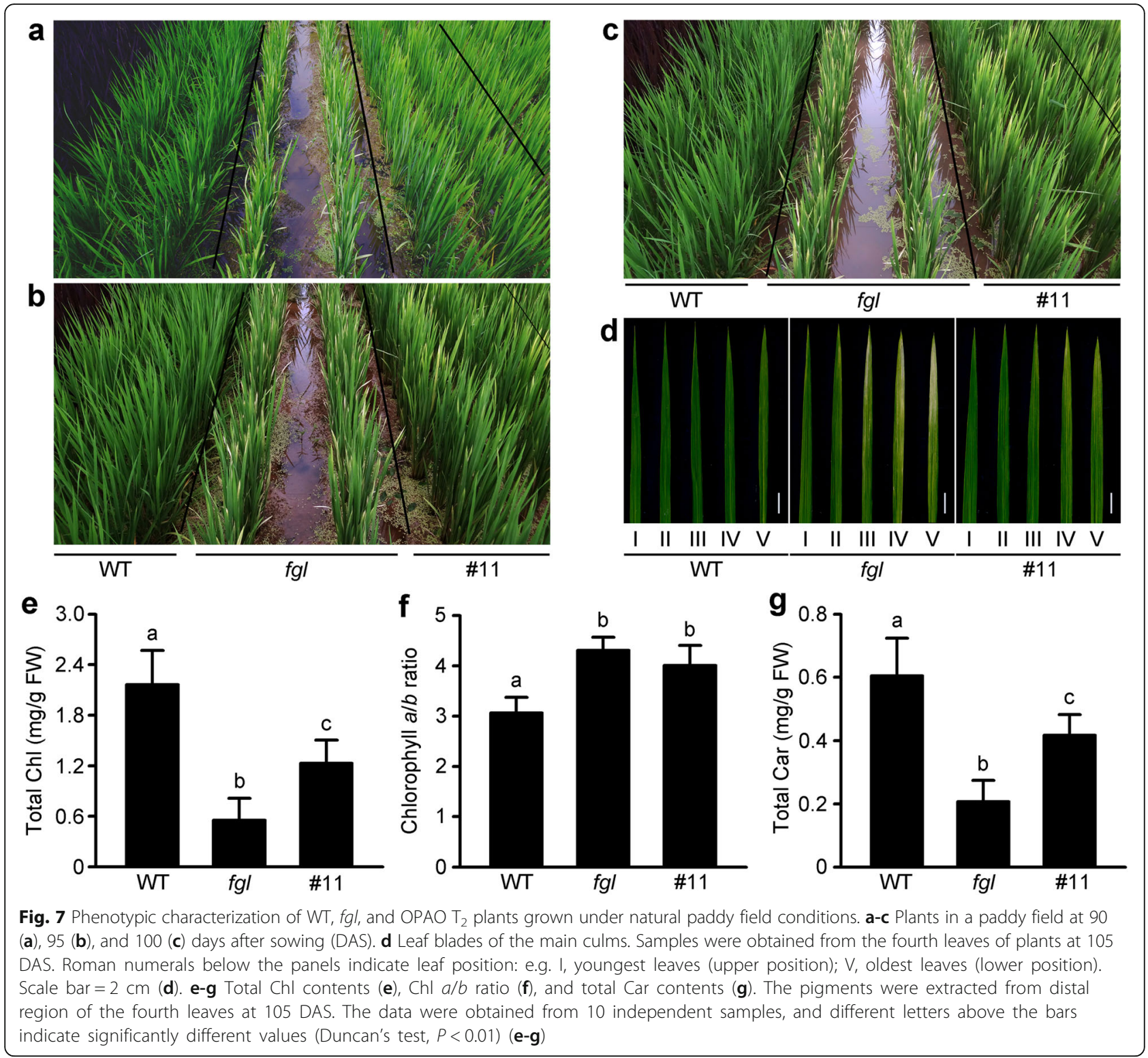

(Sakuraba et al. 2013). In particular, the levels of OsPORA mRNA and protein are rapidly downregulated under long periods and high levels of illumination (Sakuraba et al. 2013). Similarly, Arabidopsis PORA (AtPORA) mRNA is barely detectable in leaf tissues of plants grown in the light, in contrast to AtPORB and AtPORC mRNA levels (Armstrong et al. 1995; Su et al. 2001). In the OPAO lines, we predicted that OSPORA would be constitutively expressed throughout development. As expected, OsPORA transcript levels in OPAO line \#11 were consistent under both SD and CL conditions (Fig. 5b). Moreover, OsPORA protein levels did not differ between SD and CL conditions (Fig. 5c), indicating that excessive light does not affect the protein stability of OsPORA. However, Chl synthesis in the OPAO plants was not completely restored under $\mathrm{CL}$ conditions (Fig. 6d), although the levels of OsPORA protein remained high independent of light period. Hence, the compromised recovery of leaf greenness in the OPAO lines under CL conditions may be due to the weakening of OsPORA activity upon exposure to excessive light, and thus, the overall POR activity drops below the threshold level, which is not sufficient for producing Pchlide for Chl synthesis in the absence of light-stable OsPORB activity.

Many studies have investigated the possibility of the functional redundancy of the POR isoforms, as their exact functions in plant development are currently unclear, although their sequences are highly conserved (Sperling et al. 1998; Franck et al. 2000; Masuda et al. 2003; Frick et al. 2003; Paddock et al. 2010; Paddock et al. 2012). The amino acid sequences of OsPORA and 
OsPORB, the two POR isoforms in rice, are remarkably similar, and POR enzymes are highly conserved among angiosperms (Sakuraba et al. 2013). Because of this sequence conservation, we hypothesized that the two rice POR proteins are functionally equivalent, despite their distinct expression patterns. The Arabidopsis atporB atpoc $C$ double mutants are totally recovered by ectopic expression of AtPORA (Paddock et al. 2010). In the current study, however, overexpressing OsPORA in plants lacking OsPORB gave incomplete rescue of leaves, but only under $\mathrm{CL}$ conditions at the early vegetative stage and beginning at the early reproductive phase under natural field conditions (Figs. 4 and 7). Both lesion formation and degreening in the $f g l$ mutant under natural field conditions (beginning at the tips of older leaves) are caused by the suppression of OsPORA expression, resulting in the complete absence of POR activity (Sakuraba et al. 2013). In this study, however, we found that in developing leaves under excessive light conditions and in mature leaves beginning at the reproductive stage, even high levels of OsPORA mRNA and protein failed to fully substitute for OsPORB activity, which is required for normal levels of Chl synthesis.

\section{Conclusions}

OsPORs, which are essential for Chl synthesis, are present in two isoforms in rice, but the biological diversity of their roles has been unclear. We generated transgenic plants to investigate the functional differences between OsPORA and OsPORB under various growth conditions. Although the constant presence of OsPORA activity restored the leaf phenotype of $f g l$ mutant, the function of OsPORB was not fully replaced by OsPORA activity under certain growth conditions. Taken together, our findings indicate that the functional differentiation of the two rice PORs is closely associated with maintaining the photosynthetic activity of leaves until senescence. Thus, we propose that the two OsPORs have differentiated during evolution to play distinct roles in the adaptation of rice to the environment.

\section{Methods}

\section{Plant Materials and Growth Conditions}

The single recessive $f g l$ mutant was previously generated from japonica rice cultivar 'Kinmaze' by methyl nitrosourea (MNU) mutagenesis as previously described (Iwata and Omura 1975). For phenotypic characterization, plants were grown under natural long days ( $14 \mathrm{~h}$ light/day) in a paddy field (Suwon, Republic of Korea, $37^{\circ} \mathrm{N}$ latitude), greenhouse (Seoul, Republic of Korea), and growth chambers. The chamber conditions were: $24 \mathrm{~h}$ light at $30{ }^{\circ} \mathrm{C}$ for $\mathrm{CL}$ and $10 \mathrm{~h}$ light at $30{ }^{\circ} \mathrm{C} / 14 \mathrm{~h}$ dark at $24{ }^{\circ} \mathrm{C}$ for SD with $70 \%$ relative humidity. Light-emitting diodes (LEDs) were used and the average photon flux density was $200 \mu \mathrm{mol} \mathrm{m}^{-2} \mathrm{~s}^{-1}$.

\section{Vector Construction and Rice Transformation}

OsPORA cDNA was obtained by reverse-transcription polymerase chain reaction (RT-PCR) with total RNA extracted from the leaves of japonica rice cultivar 'Nipponbare' using gene-specific primers (Additional file 6: Table S1). OsPORA was ligated into the $\mathrm{pCR} 8 / \mathrm{GW} /$ TOPO plasmid (Invitrogen). To overexpress OsPORA in $f g l$ mutant, full-length OsPORA cDNA was subcloned into the pMDC32 Gateway binary vector containing the cauliflower mosaic virus $35 \mathrm{~S}$ promoter (Curtis and Grossniklaus 2003). The recombinant plasmid was introduced into calli generated from mature embryos from $f g l$ mutant seeds through Agrobacterium (strain EHA105)mediated transformation (Jeon et al. 2000). Transgenic plants were grown in Murashige and Skoog medium for 2 weeks and transferred to soil. Vector insertion in the transgenic plants was confirmed by PCR with primers for the pMDC32 vector and OsPORA fragment (Additional file 6: Table S1).

\section{Measurement of Photosynthetic Pigments}

To evaluate total chlorophyll and carotenoid concentrations, pigments were extracted from leaves with $80 \%$ acetone. Chlorophyll and carotenoid levels were measured with a UV/VIS spectrophotometer (BioTek) as described previously (Lichtenthaler 1987). Total Pchlide was extracted from leaf blades with $80 \%$ acetone containing $0.1 \mathrm{~N}$ ammonium hydroxide at $4{ }^{\circ} \mathrm{C}$. Total Pchlide was measured in etiolated seedlings grown in the dark for 10 days. Photoactive Pchlide levels were calculated based on the difference between total Pchlide and non-photoactive Pchlide, which was determined in illuminated etiolated seedlings. Pchlide and photoactive Pchlide concentrations were calculated using a Cary Eclipse fluorescence spectrophotometer (Varian) or UV/ VIS spectrophotometer (BioTek). The slit widths of both excitation and emission in the fluorescence spectrophotometer were set at $10 \mathrm{~nm}$, and the excitation and emission wavelengths were $433 \mathrm{~nm}$ and $634 \mathrm{~nm}$, respectively. The values from fluorescence spectrophotometry were calibrated using an absorption coefficient of $30.4 \mathrm{mM}^{-1} \mathrm{~cm}^{-1}$ (Brouers and Michel-Wolwertz 1983; Sperling et al. 1998). UV/VIS spectrophotometry was performed as described previously (Brouers and MichelWolwertz, 1983). All experiments were performed as previously described (Anderson and Boardman 1963; Brouers and Michel-Wolwertz 1983; Sperling et al. 1998; Masuda et al. 2003; Paddock et al. 2010; Paddock et al. 2012).

\section{Transmission Electron Microscopy (TEM) Analysis}

Leaf samples for TEM analysis were harvested from 3 -month-old plants grown in the greenhouse and from 10-day-old dark-grown etiolated seedlings. Whole tissue preparation was carried out as described previously (Park 
et al. 2007). Segments of leaf tissues were fixed in modified Karnovsky's fixative (2\% paraformaldehyde, $2 \%$ glutaraldehyde, and $50 \mathrm{mM}$ sodium cacodylate buffer, $\mathrm{pH}$ 7.2) and washed three times with $50 \mathrm{mM}$ sodium cacodylate buffer, $\mathrm{pH} 7.2$, at $4{ }^{\circ} \mathrm{C}$ for $10 \mathrm{~min}$. The samples were post fixed with $1 \%$ osmium tetroxide in $50 \mathrm{mM}$ sodium cacodylate buffer, $\mathrm{pH} 7.2$, at $4{ }^{\circ} \mathrm{C}$ for $2 \mathrm{~h}$ and briefly washed twice with distilled water at $25{ }^{\circ} \mathrm{C}$. The samples were en bloc stained in $0.5 \%$ uranyl acetate at $4{ }^{\circ} \mathrm{C}$ for a minimum of $30 \mathrm{~min}$, dehydrated in a gradient series of ethanol and propylene oxide, and embedded in Spurr's resin. After polymerization at $70{ }^{\circ} \mathrm{C}$ for $24 \mathrm{~h}$, the sections were sliced to $60 \mathrm{~nm}$ with an ultramicrotome (MT-X; RMC) and stained with $2 \%$ uranyl acetate for 5 min and Reynolds' lead citrate for $2 \mathrm{~min}$ at $25{ }^{\circ} \mathrm{C}$. The processed samples were then examined under a JEM-1010 EX electron microscope (JEOL).

\section{ROS Analysis}

Detection of singlet oxygen was conducted as previously described with some modifications (Sakuraba et al. 2013). For singlet oxygen staining, 3-month-old leaves were infiltrated in the dark with a solution of $200 \mu \mathrm{M}$ Singlet Oxygen Sensor Green reagent (SOSG, Invitrogen) in $50 \mathrm{mM}$ sodium potassium buffer ( $\mathrm{pH}$ 7.5). After 30 min incubation, the leaf discs were washed once with $50 \mathrm{mM}$ sodium potassium buffer and three times in distilled water. The fluorescence emission of SOSG was detected by confocal laser scanning microscopy (LSM510, Carl Zeiss). The excitation and emission wavelengths were $480 \mathrm{~nm}$ and $520 \mathrm{~nm}$, respectively. Detection of hydrogen peroxide and superoxide by 3,3-diaminobendizine (DAB) and nitroblue tetrazolium chloride (NBT) staining, respectively, was performed as previously described (Han et al. 2012).

\section{Sodium Dodecyl Sulfate Polyacrylamide Gel Electrophoresis (SDS-PAGE) and Immunoblot Analysis} Photosynthesis-related and OsPOR proteins were detected as previously described, with some modifications (Han et al. 2012; Kwon et al. 2015a). Leaf tissue (10 mg) was homogenized in $100 \mu \mathrm{l}$ of SDS sample buffer (50 mM Tris, pH 6.8, 2 mM EDTA, 10\% w/v glycerol, $2 \%$ SDS, and $6 \% \beta$-mercaptoethanol), and the extracted proteins were denatured at $100{ }^{\circ} \mathrm{C}$ for $3 \mathrm{~min}$, resolved by SDS-PAGE, and immunoblotted. All antibodies were obtained from Agrisera. Rubisco large subunit (RbcL) was visualized by staining with Coomassie Brilliant Blue reagent (Sigma-Aldrich).

\section{RT-PCR and Quantitative Reverse-transcription PCR (qRT-RCR)}

RT-PCR and qRT-PCR were carried out as previously described with slight modifications (Kwon et al. 2015b).
Total RNA was extracted from leaves using a Total RNA Extraction Kit (MGmed, Seoul, Republic of Korea). Firststrand cDNA was synthesized from $2 \mu \mathrm{g}$ total RNA using oligo $(\mathrm{dT})_{15}$ primer and M-MLV reverse transcriptase (Promega). The transcript levels of genes were detected using gene-specific primers (Additional file 6: Table S1). Glyceraldehyde phosphate dehydrogenase (GAPDH) was used as an internal control (Additional file 6: Table S1). The $20 \mu \mathrm{l}$ reactions included $2 \mu \mathrm{l}$ of $0.5 \mu \mathrm{M}$ primer, $2 \mu \mathrm{l}$ of cDNA mixture, and $10 \mu \mathrm{l}$ of $2 \mathrm{X}$ GoTaq qPCR Master Mix (Promega). PCR was performed with a Light Cycler 2.0 instrument (Roche) using the following program: $94{ }^{\circ} \mathrm{C}$ for $2 \mathrm{~min}, 40$ cycles of $94{ }^{\circ} \mathrm{C}$ for $15 \mathrm{~s}$ and $60^{\circ} \mathrm{C}$ for $1 \mathrm{~min}$.

\section{Additional files}

Additional file 1: Figure S1. PCR-based confirmation of OSPORA insertion in genomic DNA of three independent OPAO TO lines. (PDF $136 \mathrm{~kb}$ )

Additional file 2: Figure S2. Expression levels of OSPORA in the 2nd leaves of OPAO T1 lines. (PDF $125 \mathrm{~kb}$ )

Additional file 3: Figure S3. Phenotypes of $W T$, fgl mutant, and OPAO homozygous T2 line \#11 in the paddy field. (PDF $573 \mathrm{~kb}$ )

Additional file 4: Figure S4. Total Pchlide and photoactive Pchlide levels determined by UVNIS spectrophotometry. (PDF $175 \mathrm{~kb}$ )

Additional file 5: Figure S5. Phenotypic characterization of OPAO line \#11 under SD conditions. (PDF $339 \mathrm{~kb}$ )

Additional file 6: Table S1. Primers used in this study. (PDF $125 \mathrm{~kb}$ )

\section{Abbreviations}

Chl: Chlorophyll; ROS: Reactive oxygen species; Pchlide: Protochlorophyllide; Chlide: Chlorophyllide; POR: NADPH: protochlorophyllide oxidoreductase; PLB: Prolamellar body; LPOR: Light-dependent POR; DPOR: Dark-operative POR; fgl: faded green leaf; WT: Wild type; OPAO: OsPORA-overexpressing; CL: Constant light; SD: Short days; MNU: Methyl nitrosourea; LEDs: Lightemitting diodes; TEM: Transmission electron microscopy; SOSG: Singlet oxygen sensor green; DAB: 3,3-Diaminobendizine; NBT: Nitroblue tetrazolium chloride; SDS-PAGE: Sodium dodecyl sulfate polyacrylamide gel electrophoresis; RbcL: Rubisco large subunit; GAPDH: Glyceraldehyde phosphate dehydrogenase; Car: Carotenoid; CHLH: Mg-chelatase H subunit; GSAT: Glutamate-1-semialdehyde aminotransferase; DVR: 3,8-divinyl chlorophyllide a 8-vinyl reductase; Lhcb: light-harvesting Chl a/b-binding protein of photosystem II; Lhca: light-harvesting $\mathrm{Chl}$ a/b-binding protein of photosystem l; PsbC: Photosystem II protein C; PsaA: Photosystem I protein A; DAS: Days after sowing

\section{Acknowledgments}

We thank Yong-Jae Kim at the National Center for Inter-University Research Facilities (NCIRF) for technical assistance with CLSM analysis and Jihyeon Choi for technical support with the Cary Eclipse fluorescence spectrophotometer. This work was carried out with the support of the Cooperative Research Program for Agriculture \& Technology Development (Project No. PJ011063), Rural Development Administration, Republic of Korea. Choon-Tak Kwon was supported by a postdoctoral fellowship from the Basic Science Research Program (NRF-2015R1A6A3A01057535) through the National Research Foundation of Korea (NRF) funded by the Ministry of Education.

\section{Authors' Contributions}

CTK and SHK designed and performed all experiments. NCP designed and supervised the project. CTK, SHK, and NCP wrote and edited the manuscript. GS assisted in analyzing the phenotypes of transgenic plants grown under various conditions. DK helped generate the transgenic plants and analyze the phenotypes of the transgenic lines. All authors read and approved the final manuscript. 


\section{Competing Interests}

The authors declare that they have no competing interests.

Received: 16 November 2016 Accepted: 29 December 2016 Published online: 11 January 2017

\section{References}

Anderson JM, Boardman NK (1963) Studies on the greening of dark-grown bean plants. Aust J Biol Sci 17:93-101

Apel K, Santel HJ, Redlinger TE, Falk H (1980) The protochlorophyllide holochrome of barley (Hordeum vulgare L.). Isolation and characterization of the NADPH:protochlorophyllide oxidoreductase. Eur J Biochem 111:251-258

Armstrong GA, Runge S, Frick G, Sperling U, Apel K (1995) Identification of NADPH:protochlorophyllide oxidoreductases A and B: a branched pathway for light-dependent chlorophyll biosynthesis in Arabidopsis thaliana. Plant Physiol 108:1505-1517

Barber J, Morris E, Buchel C (2000) Revealing the structure of the photosystem II chlorophyll binding proteins, CP43 and CP47. Biochim Biophys Acta 1459:239-247

Benli M, Schulz R, Apel K (1991) Effect of light on the NADPH-protochlorophyllide oxidoreductase of Arabidopsis thaliana. Plant Mol Biol 16:615-625

Brouers M, Michel-Wolwertz MR (1983) Estimation of protochlorophyll(ide) contents in plant extracts; re-evaluation of the molar absorption coefficient of protochlorophyll(ide). Photosynth Res 4:265-270

Buhr F, El Bakkouri M, Valdez O, Pollmann S, Lebedev N, Reinbothe S, Reinbothe C (2008) Photoprotective role of NADPH:protochlorophyllide oxidoreductase A. Proc Natl Acad Sci U S A 105:12629-12634

Chakraborty N, Tripathy BC (1992) Involvement of singlet oxygen in 5aminolevulinic acid-induced photodynamic damage of cucumber (Cucumis sativus L.) chloroplasts. Plant Physiol 98:7-11

Curtis MD, Grossniklaus U (2003) A gateway cloning vector set for highthroughput functional analysis of genes in planta. Plant Physiol 133:462-469

Darrah PM, Kay SA, Teakle GR, Griffiths WT (1990) Cloning and sequencing of protochlorophyllide reductase. Biochem J 265:789-798

Engdahl S, Aronsson H, Sundqvist C, Timko MP, Dahlin C (2001) Association of the NADPH:protochlorophyllide oxidoreductase (POR) with isolated etioplast inner membranes from wheat. Plant J 27:297-304

Forreiter C, Apel K (1993) Light-independent and light-dependent protochlorophyllide-reducing activities and two distinct NADPHprotochlorophyllide oxidoreductase polypeptides in mountain pine (Pinus mugo). Planta 190:536-545

Forreiter C, Cleve B, Schmidt A, Apel K (1991) Evidence for a general lightdependent negative control of NADPH-protochlorophyllide oxidoreductase in angiosperms. Planta 183:126-132

Franck F, Sperling U, Frick G, Pochert B, van Cleve B, Apel K, Armstrong GA (2000) Regulation of etioplast pigment-protein complexes, inner membrane architecture, and protochlorophyllide alpha chemical heterogeneity by lightdependent NADPH:protochlorophyllide oxidoreductases A and B. Plant Physiol 124:1678-1696

Frick G, Su Q, Apel K, Armstrong GA (2003) An Arabidopsis porB porC double mutant lacking light-dependent NADPH:protochlorophyllide oxidoreductases $\mathrm{B}$ and $\mathrm{C}$ is highly chlorophylldeficient and developmentally arrested. Plant J 35:141-153

Fusada N, Masuda T, Kuroda H, Shiraishi T, Shimada H, Ohta H, Takamiya K (2000) $\mathrm{NADPH}$-protochlorophyllide oxidoreductase in cucumber is encoded by a single gene and its expression is transcriptionally enhanced by illumination. Photosynth Res 64:147-154

Gadjev I, Vanderauwera S, Gechev TS, Laloi C, Minkov IN, Shulaev V, Apel K, Inzé D, Mittler R, Van Breusegem F (2006) Transcriptomic footprints disclose specificity of reactive oxygen species signaling in Arabidopsis. Plant Physiol 141:436-445

Griffiths WT (1978) Reconstitution of chlorophyllide formation by isolated etioplast membranes. Biochem J 174:681-692

Grossman AR, Bhaya D, Apt KE, Kehoe DM (1995) Light-harvesting complexes in oxygenic photosynthesis: diversity, control, and evolution. Annu Rev Genet 29:231-288

Han SH, Sakuraba Y, Koh HJ, Paek NC (2012) Leaf variegation in the rice zebra2 mutant is caused by photoperiodic accumulation of tetra-Cis-lycopene and singlet oxygen. Mol Cells 33:87-97

Henningsen KW (1970) Macromolecular physiology of plastids VI. Changes in membrane structure associated with shifts in the absorption maxima of the chlorophyllous pigments. J Cell Sci 7:587-621
Heyes DJ, Hunter CN (2005) Making light work of enzyme catalysis: protochlorophyllide oxidoreductase. Trends Biochem Sci 30:642-649

Holtorf H, Reinbothe S, Reinbothe C, Bereza B, Apel K (1995) Two routes of chlorophyllide synthesis that are differentially regulated by light in barley (Hordeum vulgare L.). Proc Natl Acad Sci U S A 92:3254-3258

Hopkins WG (1982) Formation of chloroplast pigments in a temperature sensitive, virescent mutant of maize. Can J Bot 60:737-740

Hopkins WG, Elfman B (1984) Temperature-induced chloroplast ribosome deficiency in virescent maize. J Hered 75:207-211

Iwamoto K, Fukuda H, Sugiyama M (2001) Elimination of POR expression correlates with red leaf formation in Amaranthus tricolor. Plant J 27:275-284

Iwata N, Omura T (1975) Studies on the trisomics in rice plants (Oryza sativa L.) III. Relation between trisomics and genetic linkage groups. Jpn J Breed 25:363-368

Jeon JS, Lee S, Jung KH, Jun SH, Jeong DH, Lee J, Kim C, Jang S, Yang K, Nam J, An K, Han MJ, Sung RJ, Choi HS, Yu JH, Choi JH, Cho SY, Cha SS, Kim SI, An G (2000) T-DNA insertional mutagenesis for functional genomics in rice. Plant J 22:561-570

Kim C, Meskauskiene R, Apel K, Laloi C (2008) No single way to understand singlet oxygen signalling in plants. EMBO Rep 9:435-439

Kwon CT, Koo BH, Kim D, Yoo SC, Paek NC (2015a) Casein kinases I and $2 a$ phosphorylate Oryza sativa pseudo-response regulator 37 (OsPRR37) in photoperiodic flowering in rice. Mol Cells 38:81-88

Kwon CT, Kim SH, Kim D, Paek NC (2015b) The rice floral repressor Early flowering1 affects spikelet fertility by modulating gibberellin signaling. Rice 8:23

Lebedev N, Timko MP (1998) Protochlorophyllide photoreduction Photosynth Res 58:5-23

Lichtenthaler HK (1987) Chlorophylls and carotenoids: pigments of photosynthetic biomembranes. Methods Enzymol 148:351-382

Masuda T, Takamiya K (2004) Novel insights into the enzymology, regulation and physiological functions of light-dependent protochlorophyllide oxidoreductase in angiosperms. Photosynth Res 81:1-29

Masuda T, Fusada N, Shiraishi T, Kuroda H, Awai K, Shimada H, Ohta H, Takamiya $\mathrm{K}$ (2002) Identification of two differentially regulated isoforms of protochlorophyllide oxidoreductase (POR) from tobacco revealed a wide variety of light- and development-dependent regulations of POR gene expression among angiosperms. Photosynth Res 74:165-172

Masuda T, Fusada N, Oosawa N, Takamatsu K, Yamamoto YY, Ohto M, Nakamura K, Goto K, Shibata D, Shirano Y, Hayashi H, Kato T, Tabata S, Shimada H, Ohta $\mathrm{H}$, Takamiya K (2003) Functional analysis of isoforms of NADPH: protochlorophyllide oxidoreductase (POR), PORB and PORC, in Arabidopsis thaliana. Plant Cell Physiol 44:963-974

Matringe M, Camadro JM, Labbe P, Scalla R (1989) Protoporphyrinogen oxidase as a molecular target for diphenyl ether herbicides. Biochem J 260:231-235

Millerd A, McWilliam JR (1968) Studies on a maize mutant sensitive to low temperature I. Influence of temperature and light on the production of chloroplast pigments. Plant Physiol 43:1967-1972

Mock H, Keetman U, Kruse E, Rank B, Grimm B (1998) Defense responses to tetrapyrrole-induced oxidative stress in transgenic plants with reduced uroporphyrinogen decarboxylase or coproporphyrinogen oxidase activity. Plant Physiol 116:107-116

Oosawa N, Masuda T, Awai K, Fusada N, Shimada H, Ohta H, Takamiya K (2000) Identification and light-induced expression of a novel gene of NADPHprotochlorophyllide oxidoreductase isoform in Arabidopsis thaliana. FEBS Lett 474:133-136

op den Camp RG, Przybyla D, Przybyla D, Ochsenbein C, Laloi C, Kim C, Danon A, Wagner D, Hideg E, Göbel C, Feussner I, Nater M, Apel K (2003) Rapid induction of distinct stress responses after the release of singlet oxygen in Arabidopsis. Plant Cell 15:2320-2332

Paddock TN, Mason ME, Lima DF, Armstrong GA (2010) Arabidopsis protochlorophyllide oxidoreductase A (PORA) restores bulk chlorophyll synthesis and normal development to a porB porC double mutant. Plant Mol Biol 72:445-457

Paddock T, Lima D, Mason ME, Apel K, Armstrong GA (2012) Arabidopsis lightdependent protochlorophyllide oxidoreductase A (PORA) is essential for normal plant growth and development. Plant Mol Biol 78:447-460

Park SY, Yu JW, Park JS, Li J, Yoo SC, Lee NY, Lee SK, Jeong SW, Seo HS, Koh HJ, Jeon JS, Park YI, Paek NC (2007) The senescence-induced staygreen protein regulates chlorophyll degradation. Plant Cell 19:1649-1664

Reinbothe S, Reinbothe C, Apel K, Lebedev N (1996) Evolution of chlorophyll biosynthesis-the challenge to survive photooxidation. Cell 86:703-705 
Reinbothe S, Gray J, Rustgi S, von Wettstein D, Reinbothe C (2015) Cell growth defect factor 1 is crucial for the plastid import of NADPH:protochlorophyllide oxidoreductase A in Arabidopsis thaliana. Proc Natl Acad Sci U S A 112:5838-5843

Rosinski J, Rosen WG (1972) Chloroplast development: fine structure and chlorophyll synthesis. Q Rev Biol 47:160-191

Runge S, Sperling U, Frick G, Apel K, Armstrong GA (1996) Distinct roles for lightdependent NADPH:protochlorophyllide oxidoreductases (POR) A and B during greening in higher plants. Plant J 9:513-523

Sakuraba Y, Rahman ML, Cho SH, Kim YS, Koh HJ, Yoo SC, Paek NC (2013) The rice faded green leaf locus encodes protochlorophyllide oxidoreductase $\mathrm{B}$ and is essential for chlorophyll synthesis under high light conditions. Plant J 74:122-133

Schoefs B, Franck F (2003) Protochlorophyllide reduction: mechanisms and evolution. Photochem Photobiol 78:543-557

Schulz R, Steinmuller K, Klaas M, Forreiter C, Rasmussen S, Hiller C, Apel K (1989) Nucleotide sequence of a cDNA coding for the NADPH-protochlorophyllide oxidoreductase (PCR) of barley (Hordeum vulgare L.) and its expression in Escherichia coli. Mol Gen Genet 217:355-361

Selstam E, Sandelius AS (1984) A comparison between prolamellar bodies and prothylakoid membranes of etioplasts of dark-grown wheat concerning lipid and polypeptide composition. Plant Physiol 76:1036-1040

Shui J, Saunders E, Needleman R, Nappi M, Cooper J, Hall L, Kehoe D, StoweEvans E (2009) Light-dependent and light-independent protochlorophyllide oxidoreductases in the chromatically adapting cyanobacterium Fremyella diplosiphon UTEX 481. Plant Cell Physiol 50:1507-1521

Solymosi K, Martinez K, Kristof Z, Sundqvist C, Boddi B (2004) Plastid differentiation and chlorophyll biosynthesis in different leaf layers of white cabbage (Brassica oleracea cv. capitata). Physiol Plant 121:520-529

Solymosi K, Smeller L, Ryberg M, Sundqvist C, Fidy J, Boddi B (2007) Molecular rearrangement in POR macrodomains as a reason for the blue shift of chlorophyllide fluorescence observed after phototransformation. Biochim Biophys Acta 1768:1650-1658

Spano AJ, He Z, Michel H, Hunt DF, Timko MP (1992) Molecular cloning, nuclear gene structure, and developmental expression of $\mathrm{NADPH}$ protochlorophyllide oxidoreductase in pea (Pisum sativum L.). Plant Mol Biol 18:967-972

Sperling U, van Cleve B, Frick G, Apel K, Armstrong GA (1997) Overexpression of light-dependent PORA or PORB in plants depleted of endogenous POR by far-red light enhances seedling survival in white light and protects against photooxidative damage. Plant J 12:649-658

Sperling U, Franck F, van Cleve B, Frick G, Apel K, Armstrong GA (1998) Etioplast differentiation in Arabidopsis: both PORA and PORB restore the prolamellar body and photoactive protochlorophyllide-F655 to the cop1 photomorphogenic mutant. Plant Cell 10:283-296

Su Q, Frick G, Armstrong G, Apel K (2001) PORC of Arabidopsis thaliana: a third light- and NADPH-dependent protochlorophyllide oxidoreductase that is differentially regulated by light. Plant Mol Biol 47:805-813

Teakle GR, Griffiths WT (1993) Cloning, characterization and import studies on protochlorophyllide reductase from wheat (Triticum aestivum). Biochem J 296:225-230

Virgin HI, Kahn A, Wettsteino DV (1963) Physiology of chlorophyll formation in relation to structural changes in chloroplasts. Photochem Photobiol 2:83-91

Yoshida K, Chen RM, Tanaka A, Teramoto H, Tanaka R, Timko MP, Tsuji H (1995) Correlated changes in the activity, amount of protein, and abundance of transcript of NADPH:protochlorophyllide oxidoreductase and chlorophyll accumulation during greening of cucumber cotyledons. Plant Physiol 109:231-238

Zavaleta-Mancera HA, Franklin KA, Ougham HJ, Thomas H, Scott IM (1999) Regreening of senescent Nicotiana leaves I. Reappearance of NADPH-protochlorophyllide oxidoreductase and light harvesting chlorophyll a/b-binding protein. J Exp Bot 50:1677-1682

\section{Submit your manuscript to a SpringerOpen ${ }^{\circ}$ journal and benefit from:}

- Convenient online submission

- Rigorous peer review

- Immediate publication on acceptance

- Open access: articles freely available online

- High visibility within the field

- Retaining the copyright to your article 